\title{
Analysis of Randomness in Mechanical Properties of Carbon Nanotubes through Atomistic Simulation
}

\author{
Qiang Lu ${ }^{*}$ and Baidurya Bhattacharya ${ }^{\dagger}$ \\ University of Delaware, Newark, DE 19716, USA
}

\begin{abstract}
Carbon nanotubes (CNTs) have generated intense interest in the research community owing in part to their extraordinary mechanical properties. Nevertheless, experiment data on CNT mechanical properties exhibit significant variability. At least a part of these variabilities is due to the randomly occurring and randomly evolving defects on these small-scale structures. It is well documented that defects such as vacancies, pentagons, heptagons, Stone-Wales (SW) pairs, heterogeneous atoms etc. are commonly present in the nanotube structure. This paper studies the role of spatial randomness of defects (vacancies and $S W$ pairs) in the randomness associated with three mechanical properties (stiffness, ultimate strength and ductility) of single-walled nanotubes (SWNTs) with the help of atomistic simulation. Two SWNT configurations - a $(6,6)$ armehair and a $(10,0)$ zigzag - with aspect ratio around 6 are chosen and displacement controlled loading at the end of the tubes is applied. A modified Morse potential is adopted to model the interatomic forces. The randomness in mechanical behavior resulting only from initial velocity distribution was found to be insignificant at room temperature. Statistics of stiffness, ultimate strength and ultimate strain of the tubes as a function of defect density were determined. Cumulative damage growth in SWNTs under cyclic loading at high temperature was also investigated.
\end{abstract}

\section{Introduction}

A. Randomness in the mechanical properties of CNTs

The report by Iijima ${ }^{1}$ of the existence of helical carbon microtubules triggered widespread interest in carbon nanotubes (CNTs). Ebbesen and Ajayan ${ }^{2}$ reported the bulk synthesis of CNTs using a variant of the standard arc-discharge technique for fullerene synthesis under a helium atmosphere. Hamada et al. ${ }^{3}$ and Saito et al. ${ }^{4,5}$ calculated the electronic properties of individual nanotubes. The Young's modulus was measured by Treacy et al. ${ }^{6}$, Wong et al. ${ }^{7}$ and Falvo et al. ${ }^{8}$. An interesting statistics ${ }^{9}$ shows an exponential growth of the number of publications on CNTs: from less than 50 in 1993 to more than 1500 in 2001 . The study of carbon nanotube (CNT) has been motivated largely due to its extraordinary electronic and mechanical properties. CNT is found to be among the most robust materials: it has high elastic modulus (order of $1 \mathrm{TPa}$ ), high strength (up to $150 \mathrm{GPa}$ ), good ductility (up to $15 \%$ max strain), flexibility to bending and buckling and robustness under high pressure $^{10-13}$. The combination of these properties makes the carbon nanotube a potentially very useful material and CNTs are now used as fibers in composites, scanning probe tips, field emission sources, electronic actuators, sensors, Li ion and hydrogen storage and other electronic devices. Also, CNTs can be coated or doped to alter their properties for further applications 9 .

\footnotetext{
* Research Assistant, Dept. of Civil and Environmental Engineering, University of Delaware, Newark, DE 19716, USA

$\dagger$ Assistant Professor, Dept. of Civil and Environmental Engineering, University of Delaware, Newark, DE 19716, USA, Member, AIAA
} 
A survey of recent studies on the elastic modulus and strength of single-walled and multi-walled carbon nanotube (SWNT and MWNT) has been reported in Ref. 14. The collected data clearly show a few features: (i) the Young's modulus of SWNT is found to range from 0.31 1.25TPa, the Young's modulus of MWNT ranges from 0.1 to $1.6 \mathrm{TPa}$; (ii) the Young's modulus may depend on chiralities, but the elastic modulus measured from tubes with similar diameters varies quite significantly; (iii) experiments roughly show a trend that Young's modulus drops quickly as the diameter of tubes increases; (iv) the strength varies a lot from about $5 \mathrm{GPa}$ to $150 \mathrm{Gpa}$; (v) last but not the least important, these mechanical properties (elastic modulus, strength and failure strain) reported from experiments and analysis show significant variation. As detailed in the next section, some of the factors that give rise to randomness in mechanical properties affect CNT electronic properties as well. An analytical understanding of these variations, their sources and how they can be controlled is essential before CNTs and CNT-based products can be considered for widespread use across industries.

The huge scatter in these properties are possibly due to the variation in tube sizes (diameter and length), chiralities, random defects distribution, local fluctuations in energies, variations in the chemical environment, errors in measurements and even different ways in defining the cross-sectional area. Effects of sizes and chiralities of nanotubes on their mechanical properties have been studied $^{7,15,16}$, however, their roles are still not very clear. Although experimental measurement techniques vary in sophistication, it is still doubtful that measurement errors are fully responsible for the huge variation. The lack of consensus in defining the cross-sectional area ${ }^{17,18}$ can be rectified with a simple multiplicative factor and is not likely to be a major source of the randomness. Therefore, a substantial part of the random effect is believed to occur from random defects and/or local energy fluctuations.

\section{B. Effects of defects on the mechanical properties CNTs}

Defects such as vacancies, metastable atoms, pentagons, heptagons, Stone-Wales (SW or 5-7-7-5) defects, heterogeneous atoms, discontinuities of walls, distortion in the packing configuration of CNT bundles, etc. are widely observed in CNTs ${ }^{19-23}$. Such defects can be the result of the manufacturing process itself: according to an STM observation of the SWNTs structure, about $10 \%$ of the samples were found to exhibit stable defect features under extended scanning ${ }^{24}$. Defects can also be introduced by mechanical loading ${ }^{25,26}$ and electron irradiation ${ }^{19}$. It is reasonable to believe that these defects are randomly distributed in CNTs.

Studies have shown that defects have significant influence on the formation as well as on the electronic and mechanical properties of $\mathrm{CNTs}^{11,22,25}$. It has also been suggested that oxygen sensitivity may be an effective metric of defect concentration in carbon nanotubes ${ }^{27}$. Pentagon and heptagon defects are believed to play key roles in the formation and deformation of CNTs. For example, with the help of pentagon and heptagons, one can build special structures based on the original hexagon lattice, such as capping, intramolecular junctions, variation in diameter or chirality, welding, coalescence, $\mathrm{x}$-junction etc. ${ }^{22}$.

Vacancies result from missing carbon atoms in the CNT walls. That can happen when CNTs are subject to irradiations. The carbon atoms might be "knocked out" by either high-energy electrons or ions, and larger holes might be introduced via oxidative purification processes ${ }^{28}$. The effects of vacancy defects on CNT properties have received considerable attention ${ }^{28-30}$, although the stability of these vacancies is still in doubt. The atoms missing a neighbor have higher energy and always tend to form new bonds to reduce energy. According to simulation studies ${ }^{28}$, reconstruction happens for armchair and zigzag tubes after one or two atoms are removed. In the same study, it was found that one- and two-atom vacancy defects could reduce failure stresses by as much as $26 \%$ and markedly reduce failure strains, and large holes greatly reduce strength.

The Stone-Wales (SW) defect is composed two pentagon-heptagon pairs, and can be formed by rotating a $\mathrm{sp}^{2}$ bond by 90 degrees (SW rotation, Figure 1). SW defects are stable and commonly present in carbon nanotubes 31 , and are believed to play important roles in the mechanical ${ }^{32,33}$, electronic $^{34}$, chemical ${ }^{35}$, and other properties of carbon nanotubes. For example, Chandra et al. ${ }^{33}$ found that the SW defect significantly reduced the Elastic modulus of single-walled nanotubes. Mielke et al. ${ }^{28}$ compared the role of various defects (vacancies, holes and SW defects) in fracture of carbon nanotubes, and found that various one- and two-atom vacancies can reduce the failure stresses 
by $14 \sim 26 \%$. The SW defects were also found to reduce the strength and failure strain, although their influence was less significant than vacancies and holes.

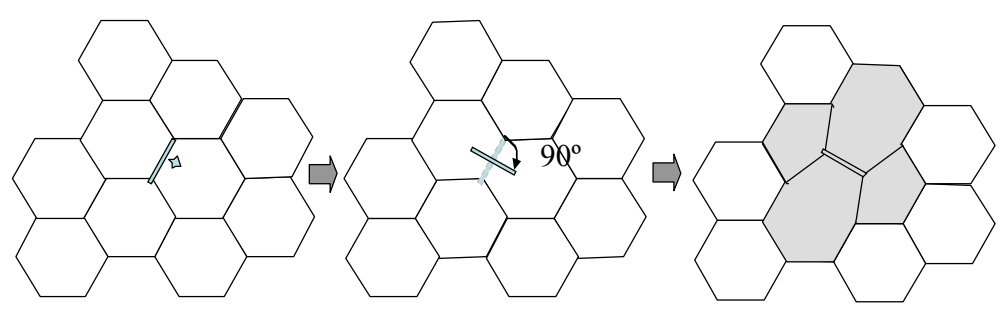

Figure 1: Formation of a Stone-Wales defect

It has been found that SWNTs, under certain conditions, respond to the mechanical stimuli via the spontaneous formation of SW defect beyond a certain value of applied strain around $5 \% \sim 6 \%{ }^{26}$. Atomistic simulations showed that these SW defects are formed when bond rotation in a graphitic network transforms four hexagons into two pentagons and two heptagons which is accompanied by elongation of the tube structure along the axis connecting the pentagons, and shrinking along the perpendicular direction. More interestingly, the SW defect can introduce successive SW rotations of different C-C bonds, which lead to gradual increase of tube length and shrinkage of tube diameter, resembling the necking phenomenon in tensile tests at macro scale. This process also gradually changes in chirality of the CNT, from armchair to zigzag direction. This whole response is plastic, with necking and growth of a "line defect", resembling the dislocation nucleation and moving in plastic deformation of crystal in many ways. Yakobson ${ }^{36}$ thus applied dislocation theory and compared the brittle and ductile failure path after the nucleation of the SW defect.

The formation of SW defects due to mechanical strains has also been reported by other groups of researchers. In their atomistic simulation study, Liew et al. ${ }^{32}$ showed that $\mathrm{SW}$ defects formed at $20 \sim 25 \%$ tensile strain for single-walled and multi-walled nanotubes with chirality ranging from $(5,5)$ to $(20,20)$. The formation of SW defects explained the plastic behavior of stress-strain curve. They also predicted failure strains of those tubes to be about $25.6 \%$. A hybrid continuum/atomistic study by Jiang et al ${ }^{37}$ reported the nucleation of SW defects both under tension and torsion. The reported SW transformation critical tensile strain is $4.95 \%$, and critical shear strain is $12 \%$. The activation energy and formation energy of the SW defects formation are also studied and related to the strength of the nanotube $\mathrm{e}^{38-40}$. The nucleation of SW defects was found to depend on the tube chiralities, diameters and external conditions such as temperature.

It is important to mentioned here that the above studies ${ }^{26,32,36,37}$ are all based on the bond order potential model. Two recent studies critical of the bond order model must also be mentioned in this context. Dumitrica et al. ${ }^{41}$ argued that the energy barrier for SW defects formation at room temperature is high enough to inhibit stress-induced SW defects formation, and showed the direct bond-breaking is a more likely failure mechanism for defect-free nanotubes. A quantum mechanical study ${ }^{42}$ from the same group pointed out that the results in Ref. 26 and related works were not reliable since the potential model they used (i.e., the Bond Order model) was not capable of correctly describing breaking of the bond connecting the two pentagons in the SW pair. They ${ }^{42}$ further found that pre-existing SW defects caused successive bond breakings instead of bond rotations as reported by ${ }^{26,32,36,37}$. Nevertheless, irrespective of how SW defects are formed and grow, it is clear that they can have significant effects of the mechanical properties of carbon nanotubes.

In conclusion, it is clear that (i) defects are commonly present in CNTs, (ii) these defects may have significant effects on the mechanical and other properties of CNTs, and (iii) a wide scatter has been observed in the mechanical properties of carbon nanotubes. Yet, surprisingly little work has been directed in the available literature toward studying the randomness in these defects and the influence of such randomness on CNT mechanical properties in a systematic and probabilistic way. In this paper we try to build toward this missing link by selecting the effect of randomly occurring vacancies and Stone-Wales defect in SWNTs. We make reasonable assumptions on the random nature of the defects and, through the technique of atomistic simulation, quantify the effect of such 
randomness on (i) the fracture process of SWNTs at the atomic scale, and (ii) three representative mechanical properties, namely, the elastic modulus, ultimate strength and ultimate strain of SWNTs.

\section{Spatial Modeling of randomly occurring defects}

An ideal modeling of material defects would require a real 3-D random field model. However, since each SW defect on a SWNT is a local rearrangement of carbon atoms on the tube wall, we can treat the SW defects as disks with a fixed area that are randomly distributed on the 2D surface. This way, it is sufficient to consider two characteristics of the defects distribution: (i) The location of the points relative to the tube surface, and (ii) the location of points relative to each other.

To our knowledge, there are few published works to date that study the effects of random defects, especially of the Stone-Wales kind, on the mechanical properties of CNTs. The study by Saether 23 investigated the transverse mechanical properties of CNT bundles subject to random distortions in their packing configuration. This distortion, quantified by a vector describing the transverse displacement of the CNTs, may be caused by packing faults or inclusions. The magnitude and direction of the vector were both uniform random variables. The transverse moduli of CNT bundles were found to be highly sensitive to small distortions in the packing configuration. In another instance, Belavin et al 30 studied the effect of random atomic vacancies on the electronic properties of CNTs. Each of the $N_{a}$ atoms in the "unit cell" was assigned (using an unspecified numbering scheme) a unique id in the range $\left[1, N_{a}\right]$. A first estimate of the number of atoms to be removed was made by generating a uniform deviate, $n_{d}$, in the interval $\left[1, N_{a}\right]$. It is not apparent how the $n_{d}$ atoms to be removed were identified. However, the atoms in this set were removed from the unit cell provided the cell was left with atoms with coordination numbers of 2 or 3 . That is, (i) the removal of an atom did not give rise to an additional dangling bond, or, (ii) if it did, the additional removal of the candidate's neighbor rectified the situation. Belavin et al's model is simple and effective for generating vacancies, but its physical basis as a $2 \mathrm{D}$ spatial random field model is not clear and it cannot be extended to areal defects involving more than one atom such as a SW defect.

Since there is not enough information in the experimental literature to provide a clear picture of statistical properties of the defects, it is reasonable to start with the assumption that the defects occur in a completely random manner, which implies an underlying homogeneous Poisson spatial process. Thus we assume vacancies to occur on the surface of the tube according to a 2-D homogeneous Poisson with constant rate $\lambda$.

For the spatial modeling of SW defects, we incorporate the additional fact that the SW defect is not a point defect but has a finite area and there should be no overlap between neighboring defects. Therefore, we adopt a Matern hard-core point process ${ }^{43}$ for the defect field. A Matern hard-core process is simply a thinned Poisson point process in which the constituent points are forbidden to lie closer than a minimum distance $h$. The intensity (i.e., rate of occurrence) of the Matern hard-core process is $\lambda_{h}=p_{h} \lambda$ where $\lambda$ is intensity of the underlying homogeneous Poisson point process and $p_{h}$ is the probability that an arbitrary point from the underlying Poisson process will survive the Matern thinning. The average number of SW defects on an area $A_{t}$ is thus $\lambda_{h} A_{t}$. For a finite tube of length $b$, the probability $p_{h}$ can be computed as:

$$
p_{h}=\frac{1}{b} \int_{0}^{b} \frac{1-e^{-\lambda A(y)}}{\lambda C(y ; h)} d y
$$

where $C$ is the area over which a Poisson point at $\left(x_{0}, y_{0}\right)$ searches for its neighbors ${ }^{44}$. In this study, we fix $h$ at $8.0 \AA$, and use a set of reasonable values for $\lambda$.. An example of an armchair SWNT with six SW defects is shown in Figure 2. 


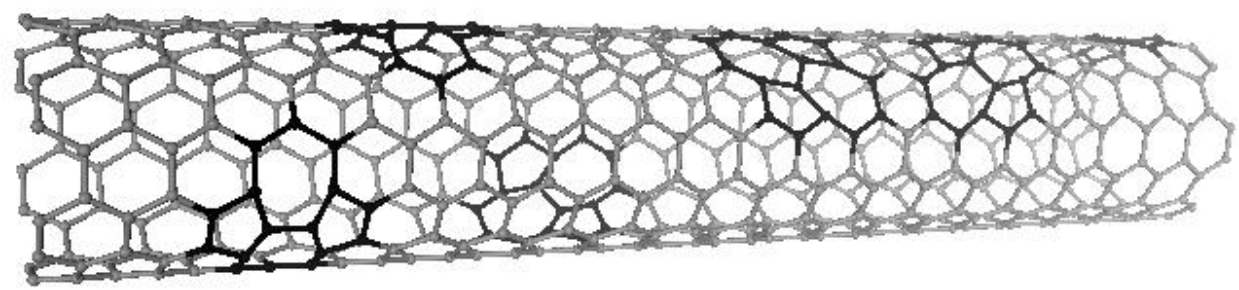

Figure 2: An armchair SWNT with six SW defects

\section{Mechanical behavior of SWNTs with random defects}

Due to the small size of carbon nanotubes, laboratory experiments to measure their mechanical properties are difficult and potentially expensive at the current state of the art. Atomistic simulation or AS, therefore, is a very attractive tool that can complement laboratory experiments in studying the mechanical behavior and failure of small scale structures such as carbon nanotubes, particularly when heterogeneities and the discrete nature of the material becomes dominant ${ }^{45}$. A modified Morse potential model for describing the interaction among carbon atoms ${ }^{46}$ is applied. This potential model does not have some of the shortcomings of the bond order potential models, for example, the cutoff function in Brenner's bond order model is said to give rise to spurious forces and inaccurately large breaking strain ${ }^{42,47,48}$.

We adopt two single-walled nanotubes (SWNT) configurations in this study: the $(6,6)$ armchair having diameter $8.14 \AA$ and the $(10,0)$ zigzag having diameter $7.82 \AA$. The total number of atoms in the simulation was 460 for the $49.2 \AA$ long $(10,0)$ zigzag tube and 480 for the $49.2 \AA$ long $(6,6)$ armchair tube. The initial atomic positions are obtained by wrapping a graphene sheet into a cylinder along the chiral vector $\mathbf{C}_{n}=m \mathbf{a}_{1}+n \mathbf{a}_{2}$ such that the origin $(0,0)$ coincides with the point $(m, n)$. The distance between neighboring carbon atoms on the graphene sheet, $a_{0}$, is $1.42 \AA$, which is the C-C sp 2 bond length in equilibrium. The tube diameter is $d=a_{0} \sqrt{3\left(m^{2}+n^{2}+m n\right)} / \pi$.

The initial atomic velocities are randomly chosen according to a uniform distribution (between the limits -0.5 and 0.5 ) and then rescaled to match the initial temperature ( $300 \mathrm{~K}$ in this example). We assume that the nanotube is in vacuum or in air, and the heat conduction between the tube and the environment is relatively small compared to the heat accumulation from the mechanical loading at high speeds. From test runs, the temperature fluctuations in the simulations are found to be within acceptable ranges. Therefore, no temperature control is implemented in this study. The mechanical loading is uniaxial and displacement controlled: it is applied by moving the atoms at either end (12 atoms at each end, as shown in Figure 3) in the axial direction away from each other at constant speed $(10 \mathrm{~m} / \mathrm{s})$ without relaxing until the occurrence of fracture.

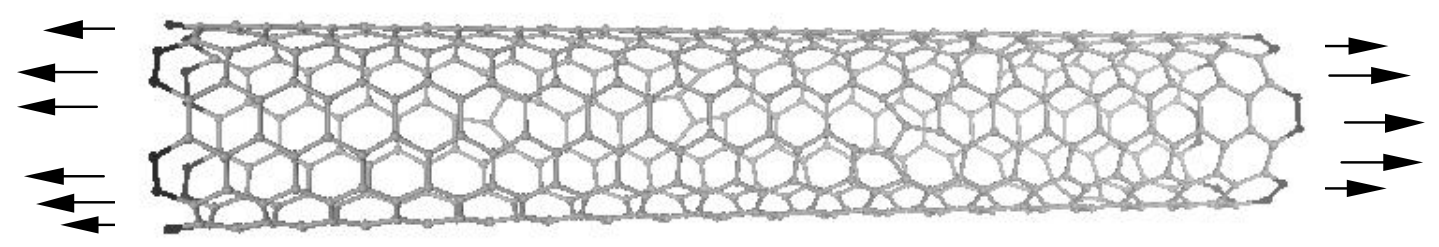

Figure 3 Mechanical loading is applied through moving the outermost atoms at both ends (highlighted at bond ends)

Three mechanical properties are calculated from the simulated force and displacement time histories: (i) the Young's modulus is calculated as the initial slope of the force-displacement curve; 
(ii) the ultimate strength is calculated at the maximum force point, $\sigma_{\mathrm{u}}=F_{\max } / A$, where $F$ is the maximum axial force, $A$ is the cross section area, assuming the thickness of tube wall is $0.34 \mathrm{~nm}$; (iii) the ultimate strain, which corresponds to the ultimate strength, is calculated as $\varepsilon_{\mathrm{u}}=\Delta L_{\mathrm{u}} / L$, where $L$ is the original tube length. Other important information can also be obtained through the simulation of tensile test of SWNTs, such as the time histories of energies and bond angles, dependence of mechanical properties on diameter, chirality and loading rate, etc. ${ }^{16-18,45,49,50}$.

Examples of the force-displacement relations of a $(6,6)$ SWNT are shown in Figure 4, with the same initial velocity distribution in each case but with different numbers of $\mathrm{SW}$ defects $(0,2,4,6$, respectively). The displacement controlled loading rate is $10 \mathrm{~m} / \mathrm{s}$. The reduced units (r.u.) used in this and subsequent figures have the following physical equivalents: 1 time r.u. $=0.03526$ picosecond; 1 force r.u. $=1.602$ nano-Newton; 1 displacement r.u. $=1$ Angstrom.

It is clear from Figure 4 that defects can have significant impact on mechanical behavior of nanotubes: SWNTs with more defects are likely to break at smaller strains and have less strength as well. The force-displacement curve in each case in Figure 4 behaves almost linearly up to about half way, although there is no obvious yield point. The slope then begins to decrease up to the breaking point where an abrupt drop of force occurs. The effect of defects on the Young's modulus, however, appears much less pronounced.

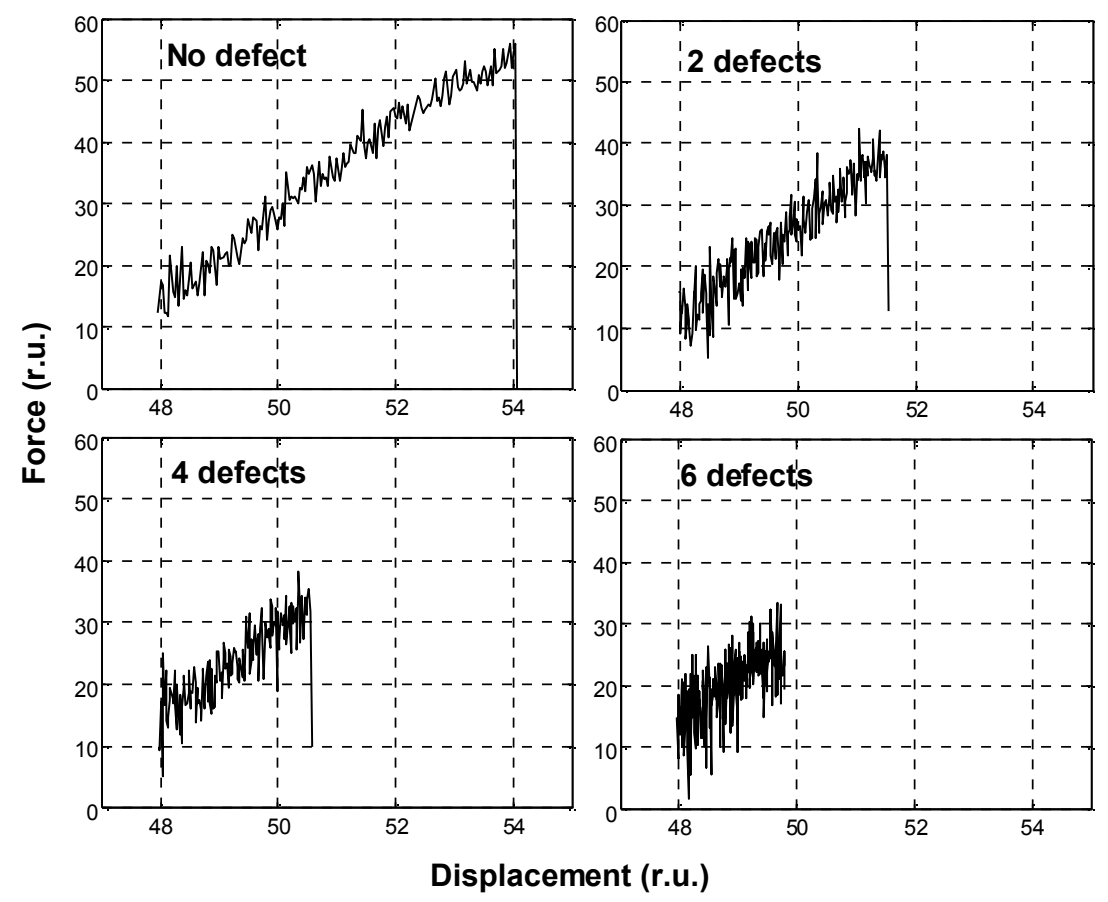

Figure 4 Force-displace ment curves of nanot ubes with various average number of $S W$ defects

We found that the crack always initiates from a defect if one exists and grows irregularly until it breaks the tube (Figure 5). For a defect-free tube, in contrast, the crack initiates at quite random locations on the tube surface. The only source of randomness in the defect free case is in the initial velocity distribution of the atoms. The tube length in each case is constant at $49.2 \AA$ as is the displacement controlled loading rate at $10 \mathrm{~m} / \mathrm{s}$. 

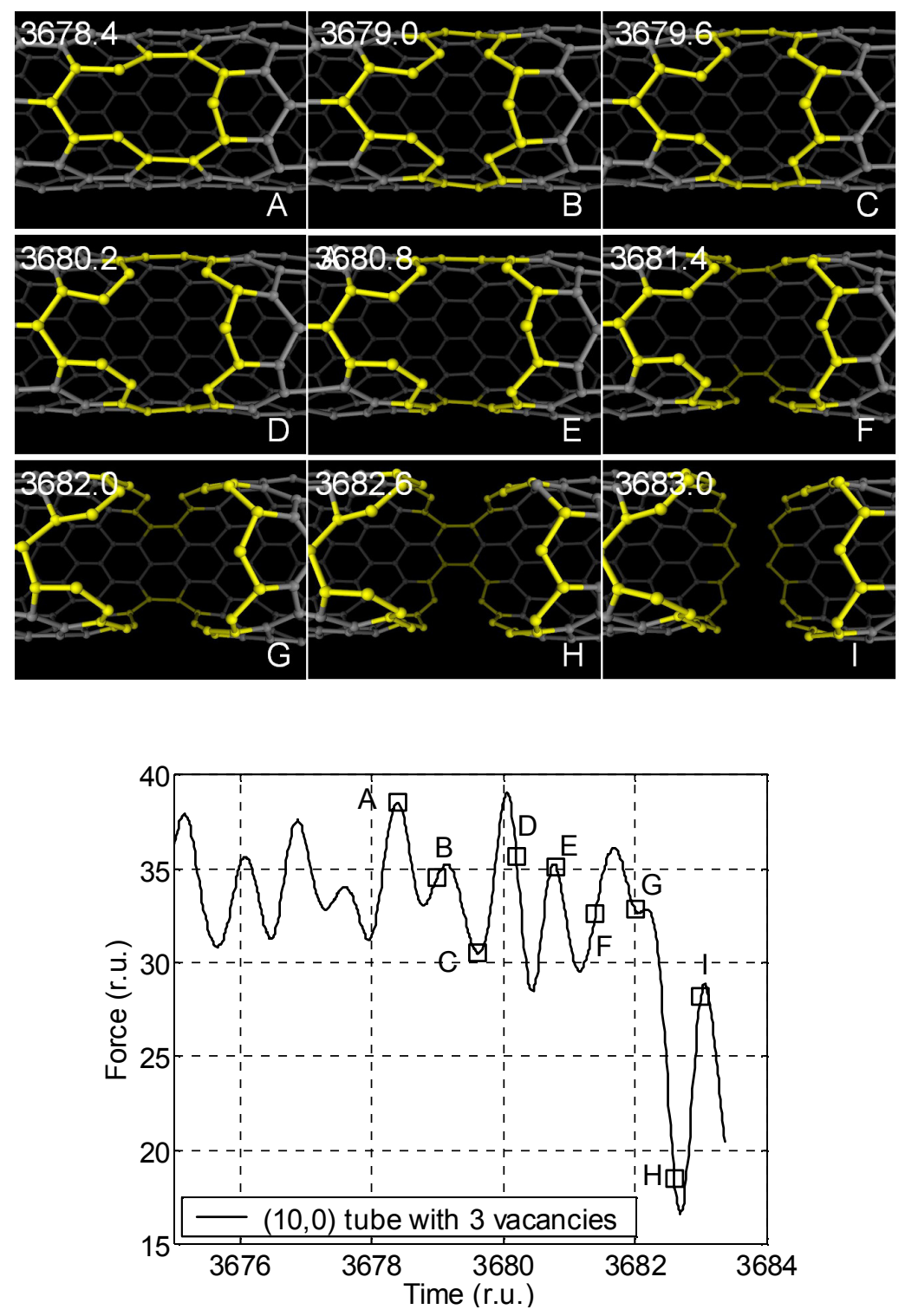

Figure 5: Fracture sequence of a $(10,0)$ zigzag tube with vacancies and corresponding force time history

The effects of randomness in the spatial distribution of SW defects and vacancies on mechanical behavior is now investigated for both the armchair and zigzag SWNTs. The tube length in each case is $49.2 \AA$ while the displacement controlled loading rate is constant at $10 \mathrm{~m} / \mathrm{s}$. For each tube, we start with the defect-free case $(\lambda=0)$ and carry out several simulations leading to fracture. From these samples we determine the statistics of the SWNT mechanical properties. The only source of randomness here is in the distribution of the initial atomic velocities. At the given temperature of $300 \mathrm{~K}$, the randomness resulting from initial velocity distribution is insignificant.

We then move on to studying (i) four cases of randomly occurring SW defects (starting from an average of 0.9 defects per tube up to 3.9 mean defects per tube) and (ii) three cases of randomly occurring vacancies (with 2, 4 and 6 mean defects per tube). In order to systematically study the effects of spatial randomness, the initial velocity distributions are kept identical to those in the respective defect-free cases. Statistical trends of the mechanical properties of the tubes with SW defects and vacancies are graphed, respectively, in Figure 6 and Figure 7. 

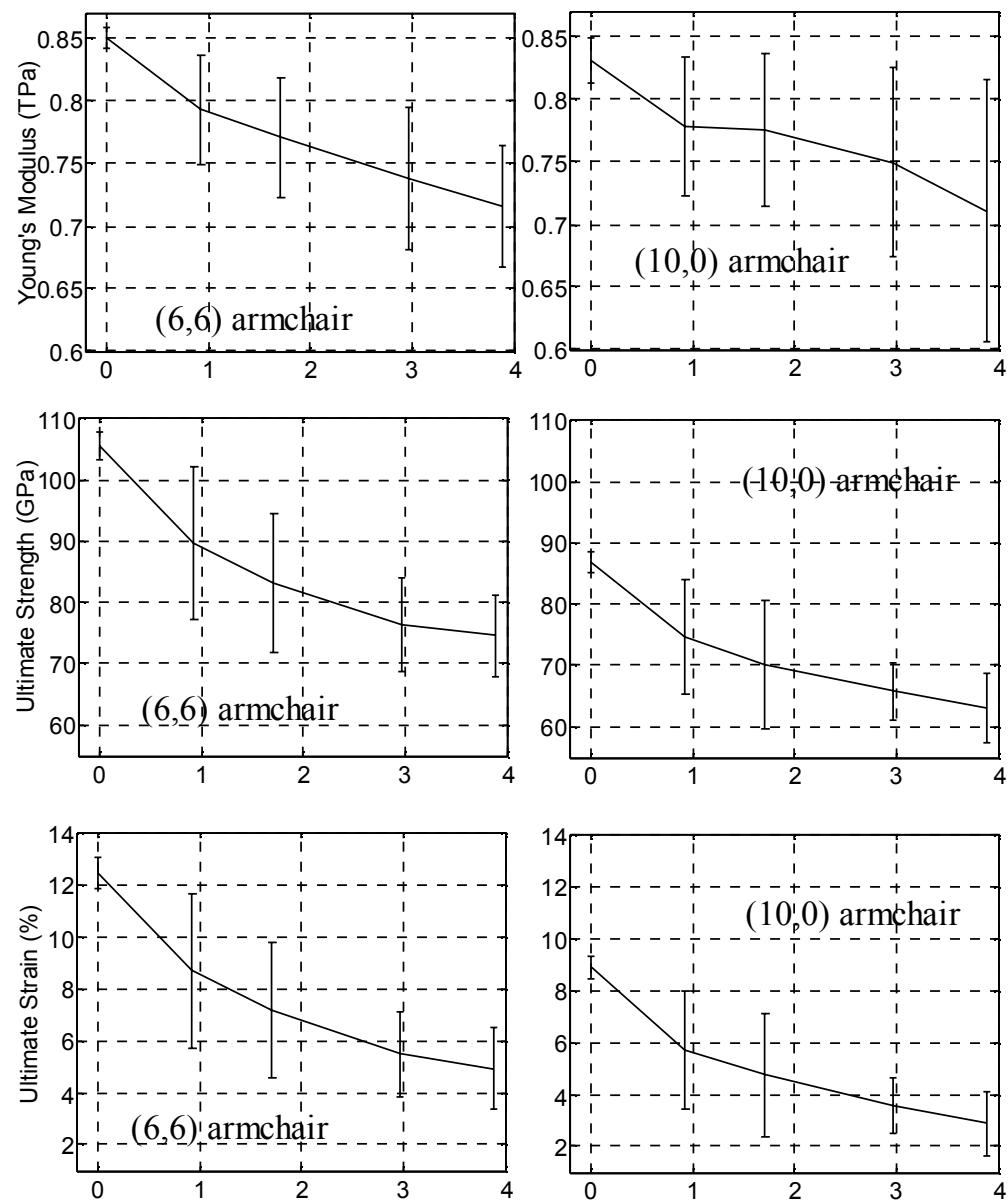

Figure 6 Effects of random SW defects on mechanical properties of armchair and zigzag SWNTs ( $l=49.2 \AA$, 50 samples each). Solid line represents mean value, vertical bar implies +/one standard deviation.

The zigzag tube differs from the armchair one in two ways. For a given mean number of defects per tube, (i) the zigzag configuration has less strength and less ductility on the average than the armchair tube although the mean stiffness is about the same; and (ii) the zigzag configuration shows more uncertainty in its stiffness and ultimate strain compared with the armchair tube although the strength has roughly the same c.o.v. in either configuration. The fact that zigzag tube has less ultimate strain has been reported by a few studies ${ }^{25,38}$ and the widely observed fact that the brittle fracture mode is more sensitive to random defects has been corroborated by the above findings.

No dislocations was observed in our simulations. This may be due to the difference in the potential models and/or the loading modes: our tensile loading is continuous and monotonic at room temperature and we used a modified Morse potential, while Nardelli et al. ${ }^{26}$ used a Bond order potential model and allowed unloading and relaxation at high temperature. 

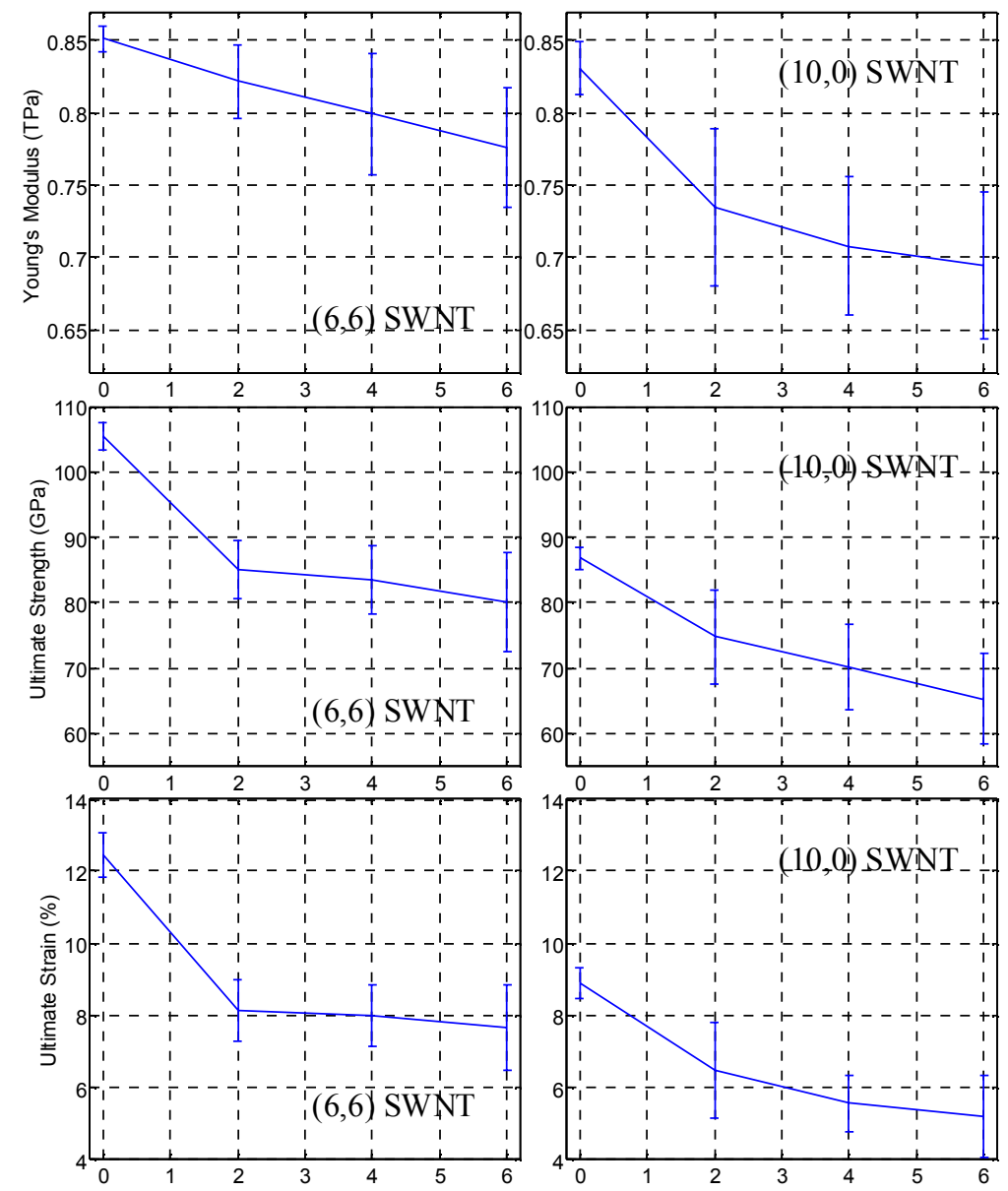

Figure 7 Effects of random vacancies on mechanical properties of armchair and zigzag SWNTs $(l=49.2 \AA, 50$ samples each). Solid line represents mean value, vertical bar implies +/- one standard deviation.

\section{Cumulative damage growth under cyclic loading at high temperature}

Although the SWNT structure is quite brittle around room temperature, its response to cyclic loading was investigated at high temperatures (up to $2000 \mathrm{~K}$ ). Temperature controlled was applied using the Anderson Thermostat ${ }^{51}$. Three armchair configurations - $(6,6),(10,10)$ and $(15,15)$ - were used for fatigue studies. Sinusoidal displacement controlled loading was applied at one end of the tube keeping the other end fixed. The minimum strain was kept at 0 , and maximum strain was varied between $1.5 \% \sim 5 \%$. The frequency of the cyclic loading ranges from $0.005745 \sim 0.598$ r.u.

In most temperature and loading ranges, carbon nanotubes broke in a brittle manner. The failure was catastrophic in these cases, once the first atomic bond broke, it was followed by successive breaking of atomic bonds and led to a complete fracture in a very short time, usually within one or two loading cycles.

However, in very few cases, cumulative damage growth was observed. For example, at 1500 Kelvin, under a strain range of 0 to $2.5 \%$ and cycling frequency of 0.0283 r.u., a Stone-Wales defect was nucleated on a $(15,15)$ tube during cyclic loading. After the formation of the defect, atomic bonds keep breaking and healing around the defect, but the defect remained stable for several cycles. Then a crack initiated from the vicinity of the defect and led to a complete fracture in a couple of cycles. Inspired by this find, a $(15,15)$ nanotube with a preexisting SW defect was put to fatigue 
simulation. Again, in most cases, the nanotube broke in a brittle manner. However, at 1500 Kelvin and strain range of $[0,1.5 \%]$, the SW defect initiates a small crack, the crack keeps stable for a few cycles and then leads to a complete fracture (Figure 8).
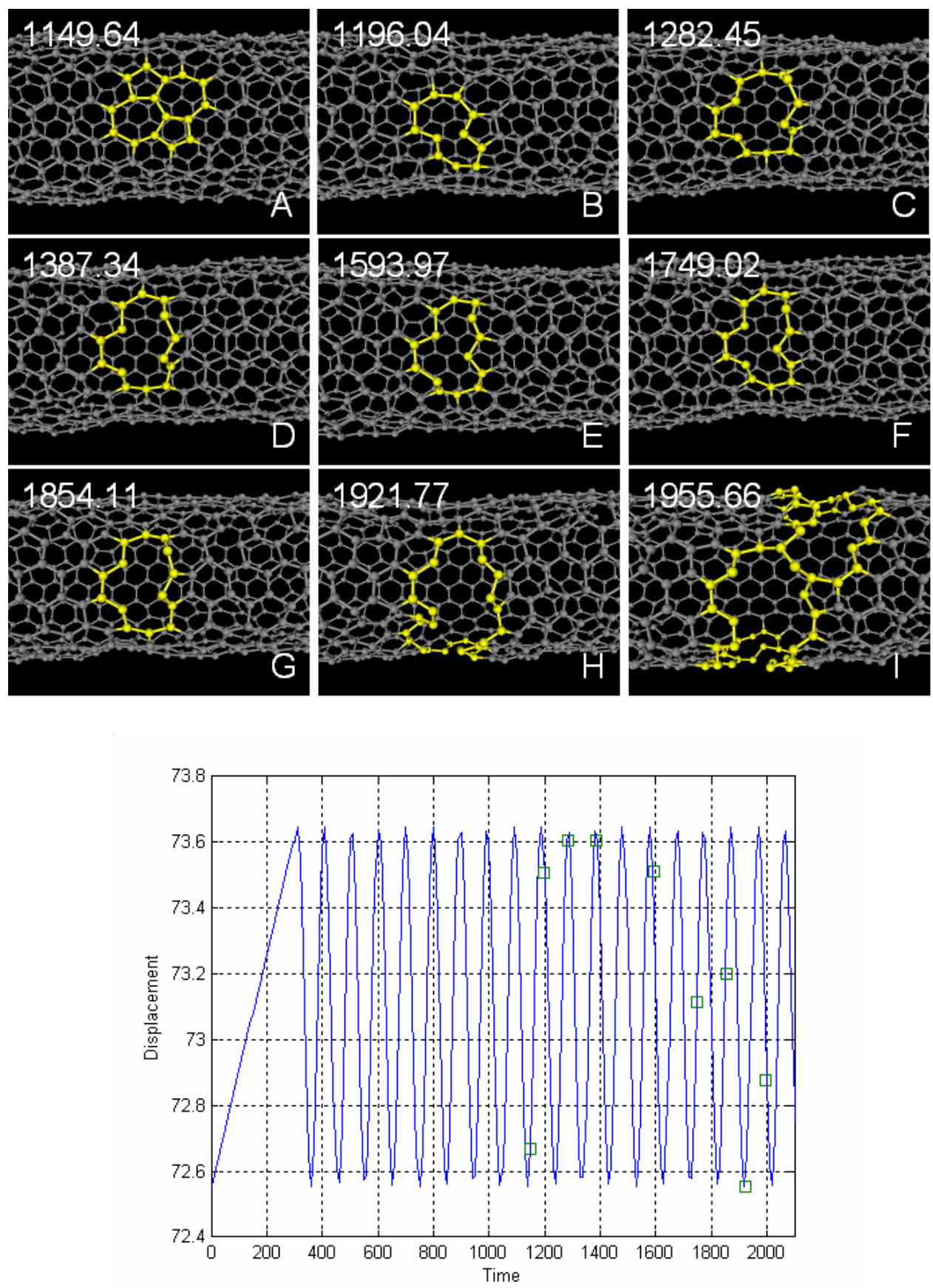

Figure 8: Cumulative damage accumulation in a $(15,15)$ SWNT at $1500 \mathrm{~K}$ under cyclic loading 


\section{Conclusions}

Carbon nanotubes are known to have ultra-high stiffness and strength, yet a wide scatter has been observed in the mechanical properties of carbon nanotubes. It is likely that defects, which are

commonly present in CNTs, may have significant effects on the mechanical and other properties of CNTs. In this paper we considered the random nature of vacancies and Stone-Wales (SW) defects in CNTs and, through the technique of atomistic simulation, demonstrated that randomly occurring defects can have significant influence on the fracture process and mechanical properties of SWNTs. A homogeneous Poisson field was adopted for the spatial distribution of vacancies, while a Matern hard-core random field applied on a finite cylindrical surface was used to describe the spatial distribution of the Stone-Wales defects. Cumulative damage growth in SWNTs with defects under load cycling at high temperature was also investigated.

\section{References}

[1] Iijima, S., Helical microtubules of graphitic carbon. Nature, 1991. 354: p. 56.

[2] Ebbesen, T.W. and P.M. Ajayan, Large-scale synthesis of carbon nanotubes. Nature, 1992. 358: p. 220.

[3] Hamada, N., S. Sawada, and A. Oshiyama, New one-dimensional conductors: Graphitic microtubules. Physical Review Letters, 1992. 68(1579-1582).

[4] Saito, R., et al., Electronic structure of graphene tubules based on C60. Physical Review B, 1992. 46: p. 1804.

[5] Saito, R., et al., Electronic structure of chiral graphene tubules. Applied Physics Letters, 1992. 60: p. 2204.

[6] Treacy, M.M., et al., Exceptional high Young's modulus observed for individual carbon nanotubes. Nature, 1996. 381(6584): p. 678.

[7] Wong, E.W., P.E. Sheehan, and C.M. Lieber, Nanobeam mechanics: Elasticity, strength, and toughness of nanorods and nanotubes. Science, 1997. 277(5334): p. 1971-1975.

[8] Falvo, M.R., et al., Bending and buckling of carbon nanotubes under large strain. Nature, 1997. 389: p. 582.

[9] Terrones, M., Science and technology of the twenty-first century: Synthesis, properties, and applications of carbon nantoubes. Annual Review of Materials Research, 2003. 33: p. 419-501.

[10] Bernholc, J., D. Brenner, and M.B. Nardelli, Mechanical and electrical properties of nanotubes. Annual Review of Materials Research, 2002. 32: p. 347-375.

[11] Yakobson, B.I. and P. Avouris, Mechanical properties of carbon nanotubes. Topics in Applied Physics, 2001. 80: p. 287-327.

[12] Ruoff, R.S. and D.C. Lorents, Mechanical and thermal-properties of carbon nanotubes. Carbon, 1995. 33(7): p. 925-930.

[13] Salvetat, J.P., J.M. Bonard, and N.H. Thomson, Mechanical properties of carbon nanotubes. Applied Physics A, 1999. 69(3): p. 255-260.

[14] Lu, Q. and B. Bhattacharya, Effect of randomly occurring Stone-Wales defects on mechanical properties of carbon nanotubes using atomistic simulation. Nanotechnology, 2005(4): p. 555-566.

[15] Poncharal, P., et al., Electrostatic deflections and electromechanical resonances of carbon nanotubes. Science, 1999. 283(5047): p. 1513-1516.

[16] Li, C.Y. and T.W. Chou, Elastic moduli of multi-walled carbon nanotubes and the effect of van der Waals forces. Composite Science and Technology, 2003. 63(11): p. 1517-1524.

[17] Hernandez, E., C. Goze, and P. Bernier, Elastic properties of C and BxCyNz composite nanotubes. Physical Review Letters, 1998. 80(20): p. 4502-4505.

[18] Sanchez-Portal, D., et al., Ab initio structural, elastic, and vibrational properties of carbon nanotubes. Physical Review B, 1999. 59(19): p. 12678-12688.

[19] Banhart, F., Irradiation effects in carbon nanostructures. Reports on Progress in Physics, 1999. 62(8): p. 1181-1221.

[20] Iijima, S., T. Ichihashi, and Y. Ando, Pentagons, heptagons and negative curvature in graphite microtubule growth. Nature, 1992. 356(776).

[21] Zhou, O., et al., Defects in Carbon Nanostructures. Science, 1994. 263(5154): p. 1744-1747. 
[22] Charlier, J.C., Defects in Carbon nanotube. Accounts of Chemical Research, 2002. 35: p. 1063-1069.

[23] Saether, E., Transverse mechanical properties of carbon nanotube crystals. Part II: sensitivity to lattice distortions. Composite Science and Technology, 2003. 63: p. 1551-1559.

[24] Ouyang, M., et al., Atomically resolved single-walled carbon nanotube intramolecular junctions. Science, 2001. 291: p. 97.

[25] Nardelli, M.B., B.I. Yakobson, and J. Bernholc, Brittle and Ductile behavior in carbon nanotubes. Physical Review Letters, 1998. 81(21): p. 4656.

[26] Nardelli, M.B., B.I. Yakobson, and J. Bernholc, Mechanism of strain release in carbon nanotube. Physical review B, 1998. 57(8): p. R4277.

[27] Collins, P.G., et al., Extreme oxygen sensitivity of electronic properties of carbon nanotubes. Science, 2000. 287: p. 1001.

[28] Mielke, S.L., et al., The role of vacancy defects and holes in the fracture of carbon nanotubes. Chemical Physics Letters, 2004. 390(4-6): p. 413-420.

[29] Ajayan, P.M., V. Ravikumar, and J.C. Charlier, Surface reconstructions and dimensional changes in single-walled carbon nanotubes. Physical Review Letters, 1998. 81(7): p. 1437.

[30] Belavin, V.V., L.G. Bulushev, and A.V. Okotrub, Modifications to the electronic structure of carbon nanotubes with symmetric and random vacancies. International Journal of Quantum Chemistry, 2004. 96(3): p. 239-246.

[31] Ebbesen, T.W. and T. Takada, Topological and SP3 defect structures in nanotubes. Carbon, 1995. 33(7): p. 973-978.

[32] Liew, K.M., X.Q. He, and C.H. Wong, On the study of elastic and plastic properties of multiwalled carbon nanotubes under axial tension using molecular dynamics simulation. Acta Materialia, 2004. 52(9): p. 2521-2527.

[33] Chandra, N., S. Namilae, and C. Shet, Local elastic properties of carbon nanotubes in the presence of Stone-Wales defects. Physical Review B, 2004. 69(9): p. 094101.

[34] Duplock, E.J., M. Scheffler, and P.J.D. Lindan, Hallmark of perfect graphene. Physical Review Letters, 2004. 92(22).

[35] Picozzi, S., et al., Ozone adsorption on carbon nanotubes: The role of Stone-Wales defects. Journal of Chemical Physics, 2004. 120(15): p. 7147-7152.

[36] Yakobson, B.I., Mechanical relaxation and "intramolecular plasticity" in carbon nanotubes. Applied Physics Letters, 1998. 72(8): p. 918.

[37] Jiang, H., et al., Defect nucleation in carbon nanotubes under tension and torsion: StoneWales transformation. Computer Methods in Applied Mechanics and Engineering, 2004. 193(30-32): p. 3419-3429.

[38] Zhao, Q., M.B. Nardelli, and J. Bernholc, Ultimate strength of carbon nanotubes: A theoretical study. Physical Review B, 2002. 65: p. 144105.

[39] Samsonidze, G.G., G.G. Samsonidze, and B.I. Yakobson, Kinetic Theory of SymmetryDependent Strength in Carbon Nanotubes. Physical Review Letters, 2002. 88: p. 065501.

[40] Zhou, L.G. and S.Q. Shi, Formation energy of Stone-Wales defects in carbon nanotubes. Applied Physics Letters, 2003. 83(6): p. 1222.

[41] Dumitrica, T., T. Belytschko, and B.I. Yakobson, Bond-breaking bifurcation states in carbon nanotube fracture. Journal of Chemical Physics, 2003. 118(21): p. 9485-9488.

[42] Troya, D., S.L. Mielke, and G.C. Schatz, Carbon nanotube fracture - differences between quantum mechanical mechanisms and those of empirical potentials. Chemical Physics Letters, 2003. 382(1-2): p. 133-141.

[43] Matern, B., Spatial Variation. 1960: Stockholm.

[44] Lu, Q., Influence of randomly occurring defects on mechanical behavior of carbon nanotubes, PhD thesis., in Civil and Environmental Engineering Department. 2005, University of Delaware: Newark, DE.

[45] Lu, Q. and B. Bhattacharya, The role of atomistic simulations in probing the small-scale aspects of fracture - a case study on a single-walled carbon nanotube. Engineering Fracture Mechanics, 2005. in press.

[46] Belytschko, T., et al., Atomistic simulations of nanotube fracture. Physical Review B, 2002. 65: p. 235430. 
[47] Belytschko, T., et al., Atomistic simulations of nanotube fracture. Physical Review B (Condensed Matter and Materials Physics), 2002. 65(23): p. 235430-8.

[48] Huhtala, M., et al., Improved mechanical load transfer between shells of multiwalled carbon nanotubes. Physical Review B, 2004. 70(4).

[49] Wei, C., K. Cho, and D. Srivastava, Tensile strength of carbon nanotubes under realistic temperature and strain rate. Physical Review B, 2003. 67: p. 115407.

[50] Yao, N. and V. Lordi, Young's modulus of single-walled carbon nanotubes. Journal of Applied Physics, 1998. 84(4): p. 1939.

[51] Anderson, H.C., Molecular dynamics at constant pressure and/or temperature. Journal of Chemical Physics, 1980. 72: p. 2384-2393. 